\title{
"I Can’t Move My Arms and Legs": A Rare Cause of Hypokalemia-Induced Quadriparesis
}

\author{
Muhammad Atique Alam Khan ${ }^{1}$, Artem Minalyan ${ }^{1}$, Iqra Iqbal ${ }^{1}$ \\ 1. Internal Medicine, Abington Hospital-Jefferson Health, Abington, USA
}

Corresponding author: Artem Minalyan, a.minalyan@gmail.com

\begin{abstract}
Hypokalemia is a relatively common electrolyte abnormality in hospitalized patients. Severe hypokalemia $(<2.5 \mathrm{mEq} / \mathrm{L})$ can lead to profound muscle weakness or paralysis, especially in the setting of acute onset of hypokalemia. Multiple mechanisms of hypokalemia have been described, such as decreased potassium intake, increased losses, and increased transcellular shift of potassium. Drugs can rarely cause hypokalemia by one of the above-mentioned mechanisms. Here, we report a case of cocaine use leading to severe hypokalemia manifesting as quadriparesis. The aggressive repletion of potassium led to a complete resolution of muscular weakness.
\end{abstract}

Review began 06/04/2021 Review ended 06/15/2021 Published 07/02/2021

\section{() Copyright 2021}

Khan et al. This is an open access article distributed under the terms of the Creative Commons Attribution License CC-BY 4.0., which permits unrestricted use, distribution, and reproduction in any medium, provided the original author and source are credited.
Categories: Emergency Medicine, Internal Medicine, Epidemiology/Public Health Keywords: quadriparesis, hypokalemia, cocaine, toxicity, potassium

\section{Introduction}

Cocaine is one of the most common causes of drug-related visits to the emergency departments in the United States [1]. Most of its important side effects are related to its hemodynamic effects (vasoconstriction leading to myocardial injury, stroke, seizures, etc.). These side effects are primarily mediated by the activation of the sympathetic nervous system. Severe hypokalemia has been reported to cause muscular weakness. Only a few cases have reported the association of cocaine ingestion leading to hypokalemiainduced muscular paralysis [2-5].

\section{Case Presentation}

A 43-year-old Caucasian male was brought to the emergency department (ED) after he was found lying on the floor for more than 24 hours because of an inability to move his extremities due to profound weakness. Reportedly, the patient did not experience loss of consciousness, seizures, or focal weakness. His past medical history was most significant for anxiety, depression, and polysubstance abuse. Two days prior to the ED arrival, the patient took a bag of cocaine and six tablets of alprazolam. In the ED, his vital signs were unremarkable. On physical examination, profound motor weakness in all four extremities was noted (power: 1/5). Sensory examination and deep tendon reflexes were normal. The patient was awake and alert. On electrocardiogram (ECG), he was noted to have widespread ST depressions and U waves (Figure 1).

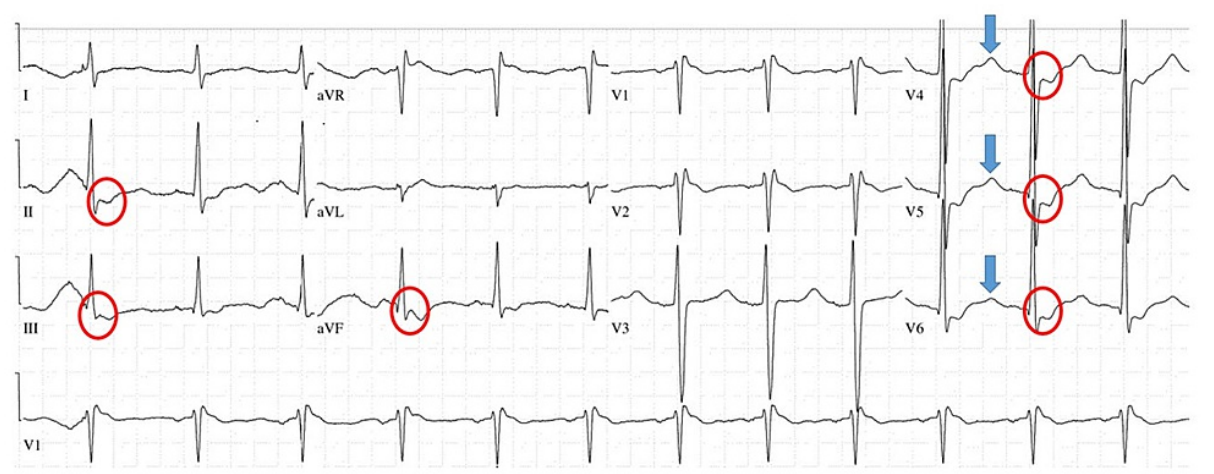

FIGURE 1: ECG demonstrating widespread ST depressions (red circles) with the presence of $U$ waves (blue arrows).

ECG: electrocardiogram 
In his labs, he was noted to have a potassium level of $1.3 \mathrm{mEq} / \mathrm{L}$, magnesium $2.7 \mathrm{mg} / \mathrm{dL}$, bicarbonate 15 $\mathrm{mEq} / \mathrm{L}$, creatine kinase $911 \mathrm{U} / \mathrm{L}$, blood urea nitrogen $16 \mathrm{mg} / \mathrm{dL}$, and creatinine $1.27 \mathrm{mg} / \mathrm{dL}$. Severe acute respiratory syndrome coronavirus 2 polymerase chain reaction was negative. Magnetic resonance imaging of the brain and cervical spine were unremarkable. The serum drug screen was also unremarkable. The urine drug screen was positive for benzodiazepines and cocaine. He was admitted to the medical intensive care unit for aggressive potassium and magnesium repletion and frequent electrolyte monitoring. Additional laboratory investigations were requested. Thyroid-stimulating hormone and cortisol levels were normal. The plasma renin-to-aldosterone ratio was slightly below normal. A spot urine potassium-to-creatinine ratio was not suggestive of urinary potassium wasting. After aggressive electrolyte repletion, the potassium started to improve (2.4 to 2.7 to 3.0). He initially got $60 \mathrm{mEq}$ of potassium chloride in the emergency department. Later, in the intensive care unit, he was given additional doses of $40 \mathrm{mEq}$ based on the repeated basic metabolic profile checks. His motor strength gradually improved. By day three of the hospitalization, no motor weakness was observed. The patient was discharged from the hospital and asked to have a close follow-up with his primary care physician. Unfortunately, the patient did not follow up with any healthcare providers after discharge.

\section{Discussion}

Hypokalemia is a common electrolyte abnormality found in hospitalized patients. Potassium is mostly stored inside the cells, primarily in muscles. The Na-K-ATPase located in the cell membrane plays a key role in regulating the transcellular shift of potassium ions. The following three mechanisms can lead to hypokalemia: (1) decreased potassium intake; (2) increased potassium loss (perspiration, gastrointestinal, and urinary losses); and (3) increased cell entry [6]. Several factors can activate the Na-K-ATPase pump and, therefore, drive potassium intracellularly (causing hypokalemia). They include the effects of insulin and catecholamines. The latter mechanisms can be explained by the activation of beta-2 adrenergic receptors that can lead to the upregulation of Na-K-ATPase pump, Na-K-2Cl cotransporter, and by increasing insulin release [7]. The other proposed mechanisms of increased potassium entry into the cells include alkalosis, hypokalemic periodic paralysis (hereditary or acquired in the setting of hyperthyroidism), and increased uptake during the production of new blood cells, hypothermia, and drug intoxications (antipsychotics, chloroquine, etc.) [8].

In patients with established hypokalemia, obtaining a thorough history is crucial (use of drugs, diarrhea, vomiting, etc.). Laboratory evaluation includes the measurement of 24-hour urinary potassium excretion or spot urinary potassium-to-creatinine ratio. Of note, the urine potassium-to-creatinine ratio of more than 13 $\mathrm{mEq} / \mathrm{g}$ of creatinine suggests urinary potassium wasting. In addition, the assessment of acid-base status should be determined in affected patients [9].

Cocaine is a well-known stimulant that acts as a triple reuptake inhibitor (serotonin-norepinephrinedopamine) [10]. It is made from the leaves of the coca plant. Due to its sympathomimetic properties, cocaine toxicity can lead to side effects targeting multiple organ systems. They include cardiovascular (cardiac ischemia, myocardial infarction, arrhythmias, cardiomyopathy), nervous (seizures, stroke, movement disorders), respiratory (rhinitis, nasal septum perforation, hemoptysis, asthma exacerbation), gastrointestinal (xerostomia, ulcers, ischemic colitis, hepatitis), renal (acute kidney injury, hypertensive nephrosclerosis), endocrine (activation of the hypothalamic-pituitary-adrenal axis), and cutaneous (pseudovasculitic lesions when contaminated with levamisole) systems [11]. The ingestion of cocaine can cause pathological changes leading to hypokalemia via several mechanisms. As a powerful stimulant, cocaine exerts its effects via sympathetic pathways which can lead to the activation of the Na-K-ATPase pump leading to hypokalemia. Alternatively, cocaine can directly target potassium channels [12].

Cocaine can also cause rhabdomyolysis via cocaine-induced vasospasm [13]. In our patient, rhabdomyolysis was not severe enough to explain the degree of muscle weakness. This supports the conclusion that severe hypokalemia was the culprit for quadriparesis. In addition, rhabdomyolysis is known to cause hyperkalemia which was not evident in our patient. Although as a stimulant, cocaine is known to cause a myriad of clinical manifestations (mostly neuropsychiatric and cardiovascular), our patient did not develop the abovementioned complications.

\section{Conclusions}

In patients presenting with muscle weakness associated with hypokalemia, the use of recreational drugs should be considered. It is important to perform a comprehensive workup when evaluating patients, including serum and urinary drug panels. Notably, cocaine toxicity can present with an isolated muscular weakness in the setting of hypokalemia while other common side effects are absent. The repletion of potassium in affected patients can lead to a complete resolution of muscular weakness.

\section{Additional Information \\ Disclosures}

Human subjects: Consent was obtained or waived by all participants in this study. Conflicts of interest: In compliance with the ICMJE uniform disclosure form, all authors declare the following: Payment/services 
info: All authors have declared that no financial support was received from any organization for the submitted work. Financial relationships: All authors have declared that they have no financial relationships at present or within the previous three years with any organizations that might have an interest in the submitted work. Other relationships: All authors have declared that there are no other relationships or activities that could appear to have influenced the submitted work.

\section{References}

1. John WS, Wu LT: Trends and correlates of cocaine use and cocaine use disorder in the United States from 2011 to 2015. Drug Alcohol Depend. 2017, 180:376-84. 10.1016/j.drugalcdep.2017.08.031

2. Shrestha R, Bhatt VR, Chaudhary RK, Sedhai R, Cowasji S: Hypokalemic quadriparesis secondary to abuse of cocaine and heroin. R I Med J (2013). 2015, 98:32-3.

3. Nalluri P, Venkatesh S, Rao A: Cocaine-induced hypokalemic paralysis. Muscle Nerve. 2000, 23:1773. 10.1002/1097-4598(200011)23:11<1773::aid-mus17>3.0.co;2-8

4. Kritikos K, Soitou K, Kapranou R, Mavroidis N: Cocaine induced severe muscular weakness. Acta Clin Belg. 2014, 69:120-1. 10.1179/2295333713Y.0000000003

5. Lajara-Nanson WA: Cocaine induced hypokalaemic periodic paralysis. J Neurol Neurosurg Psychiatry. 2002, 73:92. 10.1136/jnnp.73.1.92

6. Kardalas E, Paschou SA, Anagnostis P, Muscogiuri G, Siasos G, Vryonidou A: Hypokalemia: a clinical update . Endocr Connect. 2018, 7:R135-46. 10.1530/EC-18-0109

7. Moratinos J, Reverte M: Effects of catecholamines on plasma potassium: the role of alpha- and betaadrenoceptors. Fundam Clin Pharmacol. 1993, 7:143-53. 10.1111/j.1472-8206.1993.tb00228.x

8. Lin SH, Halperin ML: Hypokalemia: a practical approach to diagnosis and its genetic basis . Curr Med Chem. 2007, 14:1551-65. 10.2174/092986707780831050

9. Asmar A, Mohandas R, Wingo CS: A physiologic-based approach to the treatment of a patient with hypokalemia. Am J Kidney Dis. 2012, 60:492-7. 10.1053/j.ajkd.2012.01.031

10. Nestler EJ: The neurobiology of cocaine addiction. Sci Pract Perspect. 2005, 3:4-10. 10.1151/spp05314

11. Boghdadi MS, Henning RJ: Cocaine: pathophysiology and clinical toxicology. Heart Lung. 1997, 26:466-83. 10.1016/s0147-9563(97)90040-6

12. O'Leary ME, Hancox JC: Role of voltage-gated sodium, potassium and calcium channels in the development of cocaine-associated cardiac arrhythmias. Br J Clin Pharmacol. 2010, 69:427-42. 10.1111/j.13652125.2010.03629.x

13. Nolte KB: Rhabdomyolysis associated with cocaine abuse. Hum Pathol. 1991, 22:1141-5. 10.1016/00468177(91)90267-s 